\title{
Ganoderma lucidum ('Lingzhi'), a Chinese medicinal mushroom: biomarker responses in a controlled human supplementation study
}

\author{
Sissi Wachtel-Galor ${ }^{1}$, Brian Tomlinson ${ }^{2}$ and Iris F. F. Benzie ${ }^{1 *}$ \\ ${ }^{1}$ Ageing \& Health Group, School of Nursing, The Hong Kong Polytechnic University, Kowloon, Hong Kong SAR, \\ China \\ ${ }^{2}$ Division of Clinical Pharmacology, The Chinese University of Hong Kong, Hong Kong SAR, China
}

(Received 15 May 2003 - Revised 6 October 2003 - Accepted 9 October 2003)

\begin{abstract}
Lingzhi (Ganoderma lucidum) is a woody mushroom highly regarded in traditional medicine and is widely consumed in the belief that it promotes health and longevity, lowers the risk of cancer and heart disease and boosts the immune system. However, objective scientific validation of the putative health benefits of Lingzhi in human subjects is lacking, and issues of possible toxicity must be addressed. The present double-blinded, placebo-controlled, cross-over intervention study investigated the effects of 4 weeks Lingzhi supplementation on a range of biomarkers for antioxidant status, CHD risk, DNA damage, immune status, and inflammation, as well as markers of liver and renal toxicity. It was performed as a follow-up to a study that showed that antioxidant power in plasma increased after Lingzhi ingestion, and that $10 \mathrm{~d}$ supplementation was associated with a trend towards an improved CHD biomarker profile. In the present study, fasting blood and urine from healthy, consenting adults ( $n$ 18; aged 22-52 years) was collected before and after 4 weeks supplementation with a commercially available encapsulated Lingzhi preparation ( $1.44 \mathrm{~g}$ Lingzhi/d; equivalent to $13.2 \mathrm{~g}$ fresh mushroom/d) or placebo. No significant change in any of the variables was found, although a slight trend toward lower lipids was again seen, and antioxidant capacity in urine increased. The results showed no evidence of liver, renal or DNA toxicity with Lingzhi intake, and this is reassuring. The present study of the effects in healthy, well-nourished subjects provides useful, new scientific data that will support controlled intervention trials using atrisk subjects in order to assess the therapeutic effect of Lingzhi in the promotion of healthy ageing.
\end{abstract}

Lingzhi: Ganoderma lucidum: Supplementation studies: Herbal medicine

Since the beginning of human civilisation, ethnobotanicals such as herbs and mushrooms have been valued for both culinary and medicinal properties (Wasser \& Weis, 1999; Mahady, 2001). The base assumption in herbal medicine is that plants contain natural substances that can promote health and alleviate illness. There is also a general, but erroneous, assumption that natural substances and traditional remedies are non-toxic (Mahady, 2001).

During the last decade, the use of traditional medicines has expanded globally, and over-the-counter supplements have become very popular. Furthermore, this trend of increased usage of traditional remedies accelerates when conventional medicine is ineffective in the treatment of disease (Eisenberg et al. 1993, 1998). Hence, it is crucial that, along with the growing interest of the population in herbs and medicinal mushrooms, scientific research should be conducted in order to evaluate and investigate the benefits and possible deleterious effects of ethnobotanicals.

Ganoderma lucidum is a large, dark mushroom with a glossy exterior and a woody texture. Among cultivated mushrooms, Ganoderma is unique in being consumed for its medicinal, rather than nutritional value (Chang \& Buswell, 1999). It is widely consumed throughout the world as a health food, being commonly and regularly used by many Asian individuals for the promotion of health and longevity, and as a remedy for illness (Jong \& Birmingham, 1992; Rajarathnam et al. 1998; Wachtel-Galor et al. 2003). In China, G. lucidum is called 'Lingzhi'. The Japanese name for the Ganoderma family is 'Reishi'. A variety of commercial Lingzhi products is found in the forms of tea, powder and dietary supplements, and these are produced from different parts of the mushroom, predominantly the fruit body, or cap (Chang \& Buswell, 1999). These products have a significant market value, and are promoted as effective food supplements for health maintenance.

Lingzhi has long been reputed to extend the life span, and to increase youthful vigour and vitality. The specific reported attributes of Lingzhi include lowering the risk of cancer, heart disease and infection; these health-promoting effects are believed to be mediated via the antioxidant, 
hypotensive, anti-inflammatory and immunomodulatory properties of the mushroom (Willard, 1990; Chen \& Miles, 1996; Teeguarden, 1998; Wachtel-Galor et al. 2003). However, although some research has been performed on the putative health benefits of Lingzhi in animal and in in vitro models (for a review, see Wachtel-Galor et al. 2003), objective, controlled, scientific investigation of supplementation-related effects is lacking, and data on human subjects are few. It was appropriate in the present trial, therefore, to study responses in healthy subjects before attempting to assess the effects in patients or in subjects at high risk of age-related disease. The present study was planned as a follow-up to a study ( $n 10)$ of bioavailability and effects of short-term supplementation with Lingzhi (Wachtel-Galor et al. 2004). In this previous study it was found that the plasma total antioxidant level (as the ferric reducing/antioxidant power (FRAP) value; Benzie \& Strain, 1996) increased significantly within $3 \mathrm{~h}$ of a single dose of Lingzhi (Wachtel-Galor et al. 2004), indicating the absorption of antioxidants from Lingzhi. The results also were suggestive of an improved biomarker profile in terms of CHD risk after $10 \mathrm{~d}$ of Lingzhi supplementation. The aim of the present study was to further investigate the effects of Lingzhi supplementation on a wider range of biomarkers of its reported health effects, as this may provide some explanation of its reputed effects. Specifically, a range of biomarkers of antioxidant status, oxidative stress, CHD risk, inflammation and immune status was measured, as these are all relevant to age-related disease. In addition, possible toxic effects were investigated by monitoring renal and liver biomarkers in plasma.

\section{Material and methods}

\section{Subjects}

Eighteen apparently healthy Chinese adults aged between 22 and 52 years (mean 32 (SD 10) years), all non-smokers, consented to take part in the present double-blinded, crossover, placebo-controlled intervention study. On day one, a fasting heparinised blood sample and a urine sample were collected from each subject, and the blood pressure (by automated sphygmomanometer; Colin Medical Instruments Corp, Komeki City, Japan) and weight of each subject were recorded. Subjects were allocated, on a non-selective basis stratified for number, to take one of two treatments for 4 weeks. Treatments were: four capsules Lingzhi/d, or four capsules placebo/d. The dose of Lingzhi was $1.44 \mathrm{~g}$ Lingzhi extract/d, which is equivalent to $13.2 \mathrm{~g}$ fresh mushroom/d. This dosage was selected on the basis of common usage of this herbal supplement, as there is no accepted recommended daily allowance at this time. On day 29, repeat fasting blood and urine samples were collected, and blood pressure and weight recorded. There was then a 4-6 week washout period before subjects crossed over onto the other treatment. No capsules were taken during the washout period. Both Lingzhi and placebo capsules were supplied by Vita Green Health Product Co. Ltd (Hong Kong, Hong Kong SAR, China). Compliance was checked by telephone and email contact during each 4-week treatment, and by counting returned capsules.

\section{Methods}

Antioxidant status was assessed as follows: the total antioxidant power in plasma, measured by the FRAP assay (US patented; Benzie \& Strain, 1996); plasma ascorbic acid, measured by the ferric reducing/antioxidant and ascorbic acid assay, which is a modification of the FRAP assay (Benzie \& Strain, 1999); plasma $\alpha$-tocopherol was measured by HPLC (Waters Ltd, Elstree, Herts, UK) (Chirico et al. 1993; Brandt et al. 1996); lipid-standardised $\alpha$-tocopherol was calculated (Thurnham et al. 1986). The antioxidant enzymes superoxide dismutase and gluthathione peroxidase were measured in erythrocytes using commercial kit methods (Randox, Crumlin, Co. Antrim, UK) and enzyme activities were standardised to $\mathrm{Hb}$ content. FRAP, uric acid and creatinine were also measured in the urine samples. Urine creatinine was measured by the Jaffe reaction using a commercial kit (Biosystems, Barcelona, Spain) and the results were used to standardise urine FRAP values. Oxidative stress was assessed by measuring plasma malondialdehyde using the thiobarbituric acid-reactive substances reaction with organic solvent extraction and HPLC (Waters) separation (Jentzsch et al. 1996). Biomarkers of CHD risk measured included: cholesterol and triacylglycerol which were measured using commercial kit methods (BioSystems); plasma HDL-cholesterol which was measured using a phosphotungstate precipitation method (Lopes-Virella et al. 1977) and a commercial kit for cholesterol (Biosystems); plasma LDL-cholesterol concentration which was calculated using the Friedewald equation (Friedewald et al. 1972). Plasma uric acid, a purine degradation product and independent risk factor for CHD (Fang \& Alderman, 2000), was also measured, using a commercial kit (Biosystems).

Plasma high-sensitivity C-reactive protein (hsCRP), a biomarker of subclinical inflammation and reported predictor of CHD risk (Ridker, 2003), was measured using a commercial kit (Roche, Basel, Switzerland). DNA damage and resistance to a standard oxidant challenge (induced by $\mathrm{H}_{2} \mathrm{O}_{2}$ ) in leucocytes were measured, as previously described (Collins et al. 1997; Szeto \& Benzie, 2002), by the comet (single-cell gel electrophoresis) assay, a wellvalidated tool for the detection of single strand breaks in DNA in individual cells. The results are presented as percentage DNA in the comet tail, which correlates directly with DNA damage (Collins et al. 1997). Leucocyte count and $\mathrm{Hb}$ were measured using an automated electrical impedance method and a standard spectrophotometric technique (Abbott, Abbott Park, IL, USA). The effects on cellmediated immunity were investigated using a Flow cytometer (Beckman Coulter, Miami, FL, USA) in a dual colour cytometric technique with monoclonal antibodies (CD4, CD8) (Beckman Coulter) on gated lymphocytes. The results were used to identify and quantify $\mathrm{T}$ helper $(\mathrm{CD} 4+)$ and $\mathrm{T}$ cytotoxic $(\mathrm{CD} 8+)$ cells.

Plasma alanine transaminase and aspartate aminotransferase activities were measured, using commercial kits 
(Biosystems), to investigate possible hepatotoxic effects. Plasma creatinine (Biosystems) was used to detect changes in renal function.

\section{Ethical considerations}

Ethical approval for the present study was granted by the human ethics subcommittee of The Hong Kong Polytechnic University, and all procedures involving human subjects complied with the Declaration of Helsinki, as revised in 2000 .

\section{Statistical analysis}

For statistical analysis, Graphpad software was used (version 3.0; GraphPad Software Inc., San Diego, CA, USA).

One-way ANOVA for repeated measures was used to compare the results (before (day 1) and after (day 29) both placebo and Lingzhi), and the paired $t$ test was used to compare the results between days 1 and 29 of each treatment, with each subject acting as his or her own control. Variables that do not have a normal distribution (hsCRP, total $\alpha$-tocopherol and triacylglycerol) were log-transformed before analysis. Significance was sought at the $5 \%$ level.

\section{Results}

Table 1 shows the baseline (entry) level (n 18) for each variable of interest on days 1 and 29 of each treatment.
Results, analysed by repeated-measures ANOVA, showed no significant differences $(P>0.05)$ in levels at entry to either treatment, nor were any significant differences seen in responses (pre- $v$. post-treatment) to Lingzhi or the placebo. Responses (i.e. differences between days 1 and 29 of each treatment) for some of the variables are shown in Figs. 1-5. Negative values indicate a decrease during the treatment period. Fig. 1 shows the response to treatment for plasma ascorbic acid and uric acid. Fig. 2 shows the response in terms of $\alpha$-tocopherol, lipid-standardised $\alpha$-tocopherol, hsCRP and MDA. Fig. 3 shows the changes in plasma lipids. Fig. 4 shows the changes in the CD4:CD8 value and in DNA damage in lymphocytes at baseline and after a standard oxidant challenge. Fig. 5 shows the average changes in plasma and urine FRAP after $29 \mathrm{~d}$ of Lingzhi or placebo expressed as both absolute changes and as percentage of the day 1 value for each treatment. It can be seen that, while no significant differences were demonstrated in response to Lingzhi compared with the placebo for any of the variables tested, there was a trend towards lower lipid levels after Lingzhi supplementation, compared with after the placebo (Fig. 3). In addition, while no significant changes were seen in plasma FRAP, urinary FRAP increased by almost $30 \%$ after Lingzhi supplementation. No evidence of toxic effects on the liver or kidney or DNA, as assessed by plasma aspartate aminotransferase, alanine transaminase, creatinine, and comet assay results, was seen with Lingzhi supplementation. Moreover, haematological parameters $(\mathrm{Hb}$ and leucocyte count) also remained unaffected.

Table 1. Biomarker levels before and after 4 weeks supplementation with Lingzhi (Ganoderma lucidum) or placebo ( $n$ 18; fasting samples)* (Mean values and standard errors of the mean)

\begin{tabular}{|c|c|c|c|c|c|c|c|c|}
\hline & \multicolumn{4}{|c|}{ Placebo } & \multicolumn{4}{|c|}{ Lingzhi } \\
\hline & \multicolumn{2}{|c|}{ Day 1} & \multicolumn{2}{|c|}{ Day 29} & \multicolumn{2}{|c|}{ Day 1} & \multicolumn{2}{|c|}{ Day 29} \\
\hline Plasma FRAP $(\mu \mathrm{mol} / \mathrm{l})$ & 1125 & 42 & 1148 & 37 & 1151 & 45 & 1091 & 38 \\
\hline Urine FRAP (mmol/ $\mu \mathrm{mol}$ creatinine) & 434 & 52 & 411 & 43 & 329 & 37 & 415 & 74 \\
\hline Plasma ascorbic acid $(\mu \mathrm{mol} / \mathrm{l})$ & $57 \cdot 2$ & $2 \cdot 8$ & $59 \cdot 8$ & 3.7 & $59 \cdot 9$ & $5 \cdot 2$ & $56 \cdot 9$ & 4.5 \\
\hline Lipid standardised $\alpha$-tocopherol $(\mu \mathrm{mol} / \mathrm{mmol} \mathrm{TC}+\mathrm{TG})$ & 3.89 & 0.19 & 3.72 & 0.19 & 3.99 & 0.13 & $4 \cdot 19$ & 0.18 \\
\hline Plasma TC $(\mathrm{mmol} / \mathrm{l})$ & $5 \cdot 22$ & 0.24 & $5 \cdot 35$ & 0.27 & $4 \cdot 75$ & $0 \cdot 26$ & $4 \cdot 31$ & $0 \cdot 12$ \\
\hline Plasma TG (mmol/l) & 0.91 & 0.08 & 1.05 & $0 \cdot 10$ & 0.94 & $0 \cdot 10$ & 0.85 & 0.09 \\
\hline Plasma HDL-cholesterol (mmol/l) & 1.48 & 0.08 & 1.45 & 0.10 & 1.48 & $0 \cdot 10$ & 1.42 & 0.08 \\
\hline Plasma LDL-cholesterol (mmol/l) & $3 \cdot 12$ & 0.29 & 3.61 & 0.32 & $2 \cdot 76$ & 0.23 & 2.59 & 0.20 \\
\hline hsCRP (mg/l) & 0.91 & 0.23 & 0.67 & $0 \cdot 12$ & 0.83 & 0.21 & 0.87 & 0.18 \\
\hline Plasma uric acid ( $\mu \mathrm{mol} / \mathrm{l})$ & 341 & 24 & 350 & 17 & 320 & 19 & 335 & 21 \\
\hline Erythrocyte SOD (U/g Hb) & 1183 & 23 & 1187 & 28 & 1265 & 23 & 1274 & 24 \\
\hline Percentage DNA in comet tail (baseline) & $5 \cdot 5$ & 0.7 & $5 \cdot 9$ & 0.8 & $5 \cdot 6$ & 0.9 & $5 \cdot 2$ & 0.4 \\
\hline $\begin{array}{l}\text { Percentage DNA in comet tail (after } \\
15 \mu \mathrm{M}-\mathrm{H}_{2} \mathrm{O}_{2} \text { oxidant challenge) }\end{array}$ & $11 \cdot 0$ & $0 \cdot 8$ & $10 \cdot 2$ & 0.7 & $11 \cdot 2$ & 0.9 & $10 \cdot 3$ & 0.9 \\
\hline CD4:CD8 ratio & $1 \cdot 6$ & $0 \cdot 2$ & 1.5 & $0 \cdot 1$ & 1.5 & $0 \cdot 1$ & 1.6 & $0 \cdot 2$ \\
\hline Leucocytes ( $\left.\times 10^{9} / \mathrm{I}\right)$ & $6 \cdot 06$ & 0.3 & 6.59 & 0.3 & $5 \cdot 98$ & 0.3 & $6 \cdot 27$ & 0.3 \\
\hline $\mathrm{ALT}(\mathrm{U} / \mathrm{I})$ & $18 \cdot 8$ & 3.05 & $19 \cdot 2$ & 3.05 & $19 \cdot 3$ & 2.56 & $18 \cdot 4$ & 1.98 \\
\hline AST (U/I) & $26 \cdot 2$ & $1 \cdot 72$ & $28 \cdot 3$ & 1.96 & 21.9 & 1.98 & $23 \cdot 8$ & $2 \cdot 78$ \\
\hline Creatinine $(\mu \mathrm{mol} / \mathrm{l})$ & $84 \cdot 8$ & $4 \cdot 4$ & $80 \cdot 1$ & $7 \cdot 2$ & 79.4 & $7 \cdot 0$ & $80 \cdot 6$ & 5.9 \\
\hline
\end{tabular}

FRAP, ferric-reducing/antioxidant power; TC, total cholesterol; TG, triacylglycerol; hsCRP, high-sensitivity C-reactive protein; SOD, superoxide dismutase; GPx, glutathione peroxidase; MDA, malondialdehyde; ALT, alanine transaminase; AST, aspartate aminotransferase.

* For details of procedures, see p. 264 


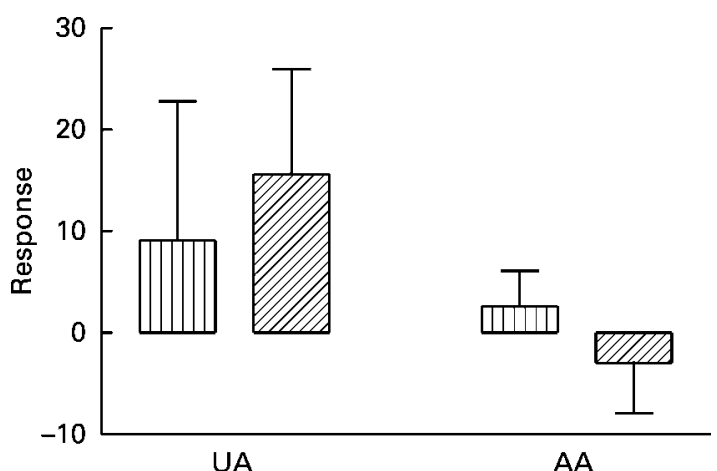

Fig. 1. Changes in fasting plasma uric acid $(U A ; \mu \mathrm{mol} / \mathrm{l})$ and ascorbic acid (AA; $\mu \mathrm{mol} / \mathrm{l})$ after $28 \mathrm{~d}$ of placebo (四) or Lingzhi (Ganoderma lucidum; $\mathbb{C}^{2}$ ). Mean values are shown for eighteen subjects, with standard errors of the mean represented by vertical bars. No statistically significant changes in response were seen $(P>0.05$; paired $t$ test)

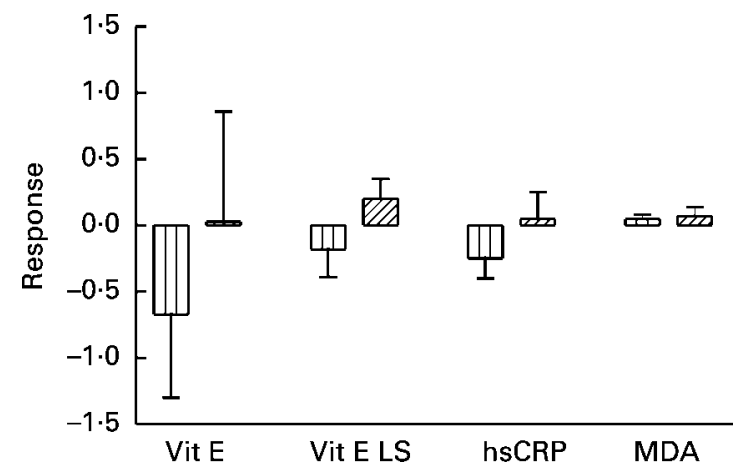

Fig. 2. Changes in fasting plasma after $28 \mathrm{~d}$ of placebo $(\mathbb{W})$ or Lingzhi (Ganoderma lucidum; 罗) of: vitamin E (as $\alpha$-tocopherol), both total (Vit E; $\mu \mathrm{mol} / \mathrm{l}$ ) and lipid-standardised (Vit E LS; $\mu \mathrm{mol} / \mathrm{mmol}$ total cholesterol + triacylglycerol); high-sensitivity Creactive protein (hsCRP; $\mathrm{mg} / \mathrm{l})$; malondialdehyde (MDA; $\mu \mathrm{mol} / \mathrm{l})$. Mean values are shown for eighteen subjects, with standard errors of the mean represented by vertical bars. No statistically significant changes in response were seen $(P>0.05$; paired $t$ test).

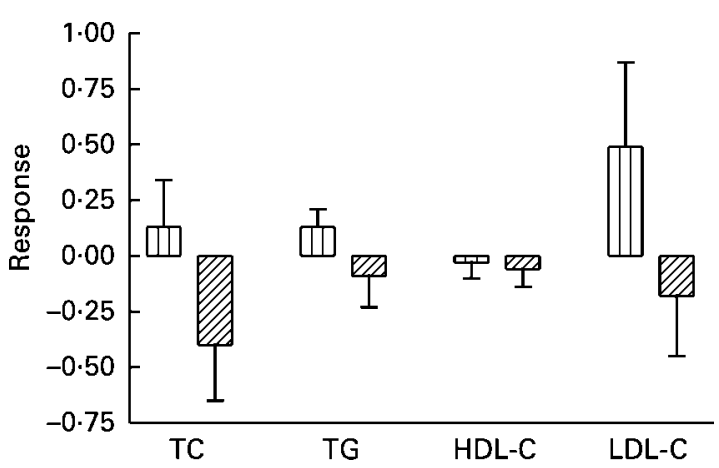

Fig. 3. Changes in total cholesterol (TC; mmol/l), triacylglycerol (TG; mmol/l), HDL-cholesterol (HDL-C; mmol/l) and LDL-cholesterol (LDL-C; $\mathrm{mmol} / \mathrm{l})$ in fasting plasma after $28 \mathrm{~d}$ of placebo $(\mathbb{W})$ or Lingzhi (Ganoderma lucidum; Mean values are shown for eighteen subjects, with standard errors of the mean represented by vertical bars. No statistically significant changes in response were seen $(P>0.05$; paired $t$ test).

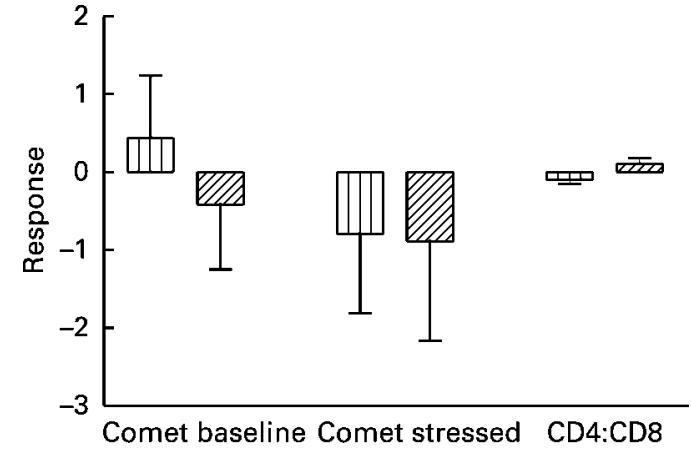

Fig. 4. Changes in lymphocyte DNA damage (as percentage DNA in the tail) without (baseline) and with (stressed) exposure to a standard oxidant challenge $\left(15 \mu \mathrm{mol} \mathrm{H}_{2} \mathrm{O}_{2}\right.$ for 5 min on ice) and the CD4:CD8 value in fasting samples after $28 \mathrm{~d}$ of placebo (四) or Lingzhi (Ganoderma lucidum; $\mathbb{Z}$ ). Mean values are shown for eighteen subjects, with standard errors of the mean represented by vertical bars. No statistically significant changes in response were seen $(P>0.05$; paired $t$ test).

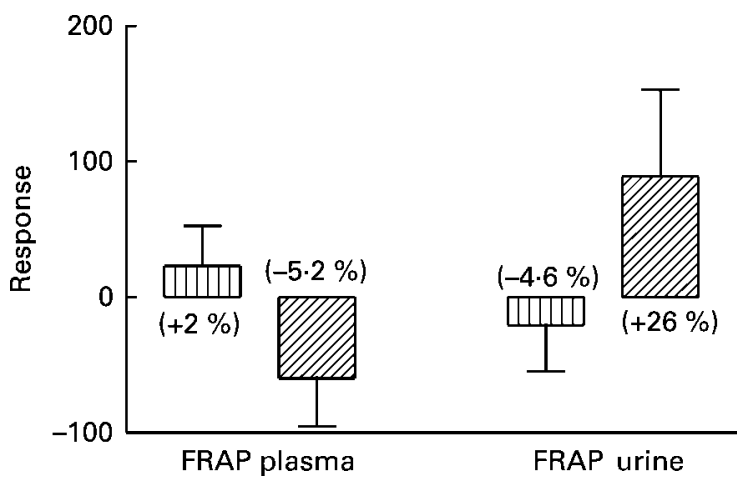

Fig. 5. Changes in total antioxidant power (as the ferric reducing/ antioxidant power (FRAP) value) in fasting plasma ( $\mu \mathrm{mol} / \mathrm{l})$ and urine $(\mathrm{mmol} / \mu \mathrm{mol}$ creatinine) after $28 \mathrm{~d}$ of placebo $(\mathbb{W})$ or Lingzhi (Ganoderma lucidum; Values in parentheses represent the change between days 1 and 29 expressed as the percentage of the day 1 value. Mean values are shown for eighteen subjects, with standard errors of the mean represented by vertical bars. No statistically significant changes in response were seen $(P>0.05$; paired $t$ test).

\section{Discussion}

In recent years, public interest in natural and alternative therapies has increased greatly in industrialised countries, with the expanding use of medicinal plants and herbal medicines. In 1990, expenditure associated with non-conventional therapy in USA was estimated at US $\$ 13.7$ billion (Eisenberg et al. 1993). This had doubled by the year 1997, with herbal medicine growing faster than any other alternative therapy (Eisenberg et al. 1998). Responding to this rapidly growing market, the National Institutes of Health have established the Center for Alternative and Complementary Medicine (National Institutes of Health, 1994), and the WHO has recognised the important contribution of traditional medicine to the provision of essential care (World Health Organization, 1991).

In addition to various parts of higher plants, mushrooms have also been used in folk medicine throughout the world 
since ancient times, and the in vitro chemical and biological properties of their fruiting bodies are numerous and well documented (Borchers et al. 1999; Wasser \& Weis, 1999). Lingzhi (G. lucidum) is a commonly used medicinal mushroom in which a wide variety of constituents, including triterpenes and polysaccharides, have been identified (Shiao et al. 1994; Chang \& Buswell, 1999). It is believed that these constituents contribute to the mushroom's antioxidant, anticancer, antiviral, and cardioprotective properties, to name but a few of the mushroom's reputed benefits. The problem facing consumers, nutritionists and other healthcare professionals now is that there are insufficient scientific data available with regard to the efficacy, safety and mode of action of Lingzhi and other traditional herbal medicines that are part of a rapidly growing market aimed at health-conscious members of the general public. In recognition of this, in March 2003, the US Food and Drug Administration announced that it will implement more stringent manufacturing and labelling standards for all dietary supplements as part of tighter quality-control measures (Food and Drug Administration, 2003). Also, in 2002 the European Parliament passed a directive on traditional herbal medicines to establish basic quality and safety standards across Europe (American Botanical Council, 2003; Medicines and Healthcare Products Regulatory Agency, 2003). This underlines the urgent need for extensive research and controlled trials to support or control the use of ethnobotanicals as food supplements for the promotion of health and functional longevity.

In the present study, the effect of 4 weeks supplementation with an encapsulated extract of the traditional 'herb' Lingzhi was determined. A wide range of biomarkers reflecting possible health benefits, in terms of age-related disease risk, as well as undesirable effects was investigated in eighteen healthy subjects in a placebo-controlled trial of cross-over design. The results indicate that Lingzhi supplementation in healthy, wellnourished subjects was not associated with toxic effects on the liver or kidney, nor was any evidence of deleterious haematological change or genotoxic effects seen. However, no statistically significant change in biomarkers of antioxidant status, CHD risk, DNA damage, immune or inflammatory status was seen. Results thus provide no clear evidence of benefit. It is possible that the lack of significant response may be related to sample size, as the study was fairly small ( $n$ 18), albeit of placebo-controlled crossover design. It is possible also to speculate that bioavailability may have played a role, and that, amongst the subjects studied, there may have been some non-responders who were responsible for the results obtained. It is relevant in this context that previous work (Wachtel-Galor et al. 2004) showed that, while all subjects studied had an increase in plasma FRAP after the ingestion of Lingzhi, there was a large variation in response.

Interestingly, no cumulative effect on plasma antioxidant status was demonstrated, although this may have been expected based on previous absorption studies, in which the plasma antioxidant capacity (as the FRAP value) significantly increased (by around $2-3 \%$ ) a few hours after Lingzhi ingestion (Wachtel-Galor et al. 2004). The apparent lack of effect of Lingzhi supplementation on total antioxidant capacity seen in the present study may be owing to an increased urinary excretion of antioxidants; however, whether these excreted antioxidants were those from Lingzhi or from other dietary or endogenous sources is not clear. It must be noted also that the apparent increase in urine FRAP after Lingzhi may have occurred because the baseline (day 1) level before Lingzhi treatment was lower than that of day 1 before placebo treatment. No significant decreases were seen in either plasma ascorbic acid or uric acid after Lingzhi supplementation, indicating no additional excretion of either of these antioxidants. The results overall are interesting, however, as they may reflect a hypothesised but as yet uncharacterised control mechanism for an integrated antioxidant defence system.

In the present study, plasma lipids did not significantly change with Lingzhi supplementation; however, a small decrease was observed in total and LDL-cholesterol and in triacylglycerol. A similar downward trend was noticed in a separate study of the effect of $10 \mathrm{~d}$ supplementation with Lingzhi (Wachtel-Galor et al. 2004). There is one published report (in Japanese with an English abstract) of a significant cholesterol-lowering effect of Lingzhi demonstrated after 6 months of supplementation. Interestingly, the Japanese study also reported a significant decrease in blood pressure in a group of essential hypertensive subjects given Lingzhi (Kanmatsuse et al. 1985). It is possible, therefore, that the putative beneficial effects of Lingzhi are more likely to be seen with a longer duration of supplementation and in those subjects with abnormal levels at entry.

Chemical studies of Lingzhi have found that polysaccharides and triterpenes are two major physiologically active constituents (Shiao et al. 1994). Triterpenes are a class of naturally occurring compounds whose $\mathrm{C}$ skeletons are composed of isoprene $\mathrm{C}_{5}$ units. In $G$. lucidum, the chemical structure of many triterpenes is similar to lanosterol (Hirotani et al. 1985), which is one of the substances in cholesterol synthesis. It is possible to speculate that Lingzhi triterpenes may have some effect on cholesterol synthesis. It is interesting to note that the natural statins, which inhibit cholesterol synthesis and are effective lipid-lowering agents, are fungal secondary metabolites (Manzoni \& Rollini, 2002). Based on this and on the present results on healthy subjects, it can be hypothesised that components of Lingzhi may possess cholesterol-lowering activities, and these may show significant beneficial effects on hyperlipidaemic subjects. A study of this kind is now being planned.

In summary, the widespread and regular use of Lingzhi, and other Chinese medicines, in health promotion is based largely on tradition rather than scientific evidence, and the issues of possible toxicity and putative benefit have not to date been adequately studied in controlled trials. In particular, human data are lacking. It was appropriate in the present trial to study responses in healthy subjects before attempting to assess the effects in patients or in subjects at high risk of age-related disease. The results of the present controlled human intervention trial are reassuring in that no evidence of liver or kidney toxicity was seen. Nor was there any evidence of genotoxic effects. However, 
no clear evidence of benefit was demonstrated in the healthy, well-nourished subjects in terms of antioxidant, immune or inflammatory status. There was no significant change in plasma antioxidant capacity after 4 weeks of Lingzhi supplementation, and the results may support a suggested control mechanism for antioxidant status. No clear hypolipidaemic effect was demonstrated, although a slight trend towards lower plasma lipids was seen, and this is worthy of further study. The new data presented here are useful and will support future studies on the effects of Lingzhi supplementation in individuals at high risk of age-related disease.

\section{Acknowledgements}

The authors are grateful to The Hong Kong Polytechnic University and to Vita Green Health Product Co. Ltd for financial support for the present study. The authors also thank Dr Y. T. Szeto for kindly sharing his expertise with the comet assay, and Mr John Yuen for his technical support during the study.

\section{References}

American Botanical Council (2003) European Union passes traditional herbal medicines directive. HerbalGram 57, 19. www.herbalgram.org

Benzie IFF \& Strain JJ (1996) The ferric reducing ability of plasma (FRAP) as a measure of 'antioxidant power': the FRAP assay. Anal Biochem 239, 70-76.

Benzie IFF \& Strain JJ (1999) Ferric reducing/antioxidant power assay: direct measure of total antioxidant activity of biological fluids and modified version for simultaneous measurement of total antioxidant power and ascorbic acid concentration. Methods Enzymol 299, 15-27.

Borchers AT, Stern JS, Hackman RM, Keen CL \& Gershwin ME (1999) Mushrooms, tumors and immunity. Proc Soc Exp Biol Med 221, 281-293.

Brandt RB, Kaugars GE, Riley WT, et al. (1996) Evaluation of serum and tissue levels of alpha-tocopherol. Biochem Mol Med 57, 64-66.

Chang ST \& Buswell JA (1999) Ganoderma lucidum (Curt.:Fr.) P. Karst. (Aphyllophoromycetideae) - a mushrooming medicinal mushroom. Int J Medicinal Mushrooms 1, 139-146.

Chen AW \& Miles PG (1996) Biomedical research and the application of mushroom nutriceuticals from Ganoderma lucidum. In Mushroom Biology and Mushroom Products, pp. 161-175 [DJ Royse, editor]. University Park, PA: Pennsylvania State University.

Chirico S, Smith C, Marchant C, Mitchinson MJ \& Halliwell B (1993) Lipid peroxidation in hyperlipidaemic patients. a study of plasma using an HPLC-based thiobarbituric acid test. Free Radic Res Commun 19, 51-57.

Collins AR, Dusinska M, Franklin M, et al. (1997) Comet assay in human biomonitoring studies: reliability, validation, and applications. Environ Mol Mutagen 30, 139-146.

Eisenberg DM, Davis RB, Ettner SL, et al. (1998) Trends in alternative medicine use in the United States, 1990-1997: results of a follow-up national survey. JAMA 280, $1569-1575$.

Eisenberg DM, Kessler RC, Foster C, Norlock F, Calkins DR \& Delbanco TL (1993) Unconventional medicine in the United States - prevalence, costs, and patterns of use. $N$ Engl J Med 328, 246-252.
Fang J \& Alderman MH (2000) Serum uric acid and cardiovascular mortality, the NHANES I epidemiologic follow-up study, 1971-1992. National Health and Nutrition Examination Survey. JAMA 283, 2404-2410.

Food and Drug Administration (2003) FDA proposes labeling and manufacturing standards for all dietary supplements. www.fda. gov/bbs/topics/news/2003/new00876.html

Friedewald WT, Levy RI \& Fredrickson DS (1972) Estimation of the concentration of low-density lipoprotein cholesterol in plasma, without use of the preparative ultracentrifuge. Clin Chem 18, 499-502.

Hirotani M, Furuya T \& Shiro MA (1985) A ganoderic acid derivative, a highly oxygenated lanostane type triterpenoid from Ganoderma lucidum. Phytochemistry 24, 2055-2059.

Jentzsch AM, Bachmann H, Furst P \& Biesalski HK (1996) Improved analysis of malondoaldehyde in human body fluids. Free Radic Biol Med 20, 251-256.

Jong SC \& Birmingham JM (1992) Medicinal benefits of the mushroom Ganoderma. Adv Appl Microbiol 37, 101-134.

Kanmatsuse K, Kajiwara N, Hayashi K, et al. (1985) Studies on Ganoderma lucidum: efficacy against hypertension and side effects (in Japanese). Yakugaku Zasshi 105, 942-947.

Lopes-Virella MF, Stone P, Ellis S \& Colwell JA (1977) Cholesterol determination in high-density lipoproteins separated by three different methods. Clin Chem 23, 882-884.

Mahady GB (2001) Global harmonization of herbal health claims. J Nutr 131, 1120S-1123S.

Manzoni M \& Rollini M (2002) Biosynthesis and biotechnological production of statins by filamentous fungi and application of these cholesterol-lowering drugs. Appl Microbiol Biotechnol 58, 555-564.

Medicines and Healthcare Products Regulatory Agency (2003) Licensing of medicines: policy on herbal medicines. www.mca.gov.uk/ourwork/licensingmeds/herbalmeds/herbal meds.htm

National Institutes of Health (1994) Exploratory Centers for Alternative Medicine Research. NIH Guide, vol. 23, no. 15, Rockville Pike, MD: National Institutes of Health.

Rajarathnam S, Shashirekha MN \& Bano Z (1998) Biodegradative and biosynthetic capacities of mushrooms: present and future strategies. Crit Rev Biotechnol 18, 91-236.

Ridker PM (2003) Clinical application of C-reactive protein for cardiovascular disease detection and prevention. Circulation 107, 363-369.

Shiao MS, Lee KR, Lin LJ \& Wang CT (1994) Natural products and biological activities of the Chinese medicinal fungus, Ganoderma lucidum. In Food Phytochemicals for Cancer Prevention. II: Teas, Spices, and Herbs. American Chemical Society Symposium Series no. 547, pp. 342-354 [CT Ho, T Osawa, MT Huang and RT Rosen, editors]. Washington, DC: American Chemical Society.

Szeto YT \& Benzie IFF (2002) Effects of dietary antioxidants on human DNA ex vivo. Free Radic Res 36, 113-118.

Teeguarden R (1998) The Ancient Wisdom of the Chinese Tonic Herbs, pp. 88-95. New York: Warner Books.

Thurnham DI, Davies JA, Crump BJ, Situnayake RD \& Davis M (1986) The use of different lipids to express serum tocopherol: lipid ratios for the measurement of vitamin E status. Ann Clin Biochem 23, 514-520.

Wachtel-Galor S, Benzie IFF, Tomlinson B \& Buswell JA (2003) Lingzhi (Ganoderma lucidum): molecular aspects of health effects. In Herbal Medicines, [L Packer, B Halliwell and CN Ong, editors]. New York: Marcel Dekker Inc. (In the Press).

Wachtel-Galor S, Szeto YT, Tomlinson B \& Benzie IFF (2004) Ganoderma lucidum ('Lingzhi'); acute and short-term 
biomarker response to supplementation. Int J Food Sci Nutr $\mathbf{5 5}$, $75-83$.

Wasser SP \& Weis AL (1999) Medicinal properties of substances occurring in higher basidiomycetes mushrooms: current perspectives (Review). Int J Medicinal Mushrooms 1, 31-62.
Willard T (1990) Reishi Mushroom: Herb of Spiritual Potency and Medical Wonder. Washington, DC: Sylvan Press.

World Health Organization (1991) Traditional medicine and modern health care. Resolution WHA44.34. www.who.int/ medicines/organization/trm/wha4434.pdf 